\title{
-NOTES-
}

\section{ROTATION OF AN INFINITE PLANE LAMINA: BOUNDARY LAYER GROWTH: MOTION STARTED IMPULSIVELY FROM REST*}

\author{
By SWAMI DAYAL NIGAM (Agra College, Agra, India)
}

1. Introduction. T. v. Kármán ${ }^{1}$ has solved the problem of rotation of an infinite plane lamina in a viscous fluid. He assumes that the motion is steady and the lamina rotates with a constant angular velocity $\Omega$ about the axis $r=0$. He has found exact solutions of the equations of motion which satisfy all the boundary conditions of the problem. The axial velocity does not vanish at infinity, but tends to a finite negative limit, which signifies a steady axial flow towards the rotating lamina. v. Kármán interprets that it is necessary to preserve continuity, since the rotating lamina acts like a centrifugal fan, the fluid moving radially outwards, especially near the lamina.

In the present note I have discussed the growth of motion in the earlier stages of its development caused by an infinite plane lamina which at $t=0$ is suddenly made to rotate with a constant angular spin $\Omega$ about the axis $r=0$. There grows a boundary layer of thickness proportional to the square root of time, adjacent to the rotating lamina which initially has a zero thickness.

We start with the equations of motion in cylindrical coordinates and substitute in them expressions for $u, v, w$ and $p$ somewhat similar to those used by v. Kármán. Then applying the approximations of the boundary layer theory, we integrate them analytically, satisfying all the boundary conditions required by the problem. The solutions have a serious limitation in that they give initial motion only. They give no information regarding the time after which the steady state is reached.

2. Equations of motion. The equations of motion in cylindrical coordinates with terms of azimuthal variation omitted are

$$
\begin{aligned}
& \nu\left[\frac{\partial^{2} u}{\partial r^{2}}+\frac{1}{r} \frac{\partial u}{\partial r}-\frac{u}{r^{2}}+\frac{\partial^{2} u}{\partial z^{2}}\right]-\frac{1}{\rho} \frac{\partial p}{\partial r}=\frac{\partial u}{\partial t}+u \frac{\partial u}{\partial r}+w \frac{\partial u}{\partial z}-\frac{v^{2}}{r} \\
& \nu\left[\frac{\partial^{2} v}{\partial r^{2}}+\frac{1}{r} \frac{\partial v}{\partial r}-\frac{v}{r^{2}}+\frac{\partial^{2} v}{\partial z^{2}}\right]=\frac{\partial v}{\partial t}+u \frac{\partial v}{\partial r}+w \frac{\partial v}{\partial z}+\frac{u v}{r} \\
& \nu\left[\frac{\partial^{2} w}{\partial r^{2}}+\frac{1}{r} \frac{\partial w}{\partial r}+\frac{\partial^{2} w}{\partial z^{2}}\right]-\frac{1}{\rho} \frac{\partial p}{\partial z}=\frac{\partial w}{\partial t}+u \frac{\partial w}{\partial r}+w \frac{\partial w}{\partial z}
\end{aligned}
$$

where $u, v, w$ and $p$ are the radial, azimuthal, and axial components of velocity and pressure respectively. The equation of continuity is

$$
\frac{\partial}{\partial r}(r u)+\frac{\partial}{\partial z}(r w)=0
$$

*Received Oct. 14, 1949.

${ }^{1}$ Goldstein, Modern developments in fluid dynamics, vol. I, The Clarendon Press, Oxford, 1938, p. 111. 
We now let

$$
\begin{aligned}
& u=\Omega^{2} r t f(\eta) \\
& v=\Omega r g(\eta) \\
& w=-4 \nu^{1 / 2} \Omega^{2} t^{3 / 2} h(\eta) \\
& p=2 \nu \rho \Omega^{2} t p(\eta)
\end{aligned}
$$

where

$$
\eta=z / 2(\nu t)^{1 / 2}
$$

During early stages of motion when $t$ is small (or in boundary layer theory terminology: when the thickness of the boundary layer is small), we may neglect the terms in the equations of motion containing higher orders of $t$. Therefore by omitting terms of order $t^{2}$ in the equations of motion and continuity, we get to a first order of approximation, the following equations ${ }^{2}$

$$
\begin{aligned}
& f^{\prime \prime}+2 \eta f^{\prime}-4 f=-4 g^{2}=-p^{\prime \prime} \\
& g^{\prime \prime}+2 \eta g^{\prime}=0 \\
& h^{\prime \prime}+2 \eta h^{\prime}-6 h=-p^{\prime} \\
& f=h^{\prime}
\end{aligned}
$$

3. Solutions of the equations. From (6) we get

$$
g=[1-\operatorname{erf} \eta]=\operatorname{erfc} \eta
$$

With this solution for $g$ the general solution of Eq. (5) may be expressed ${ }^{3}$

$f=A\left(1+2 \eta^{2}\right)+B\left[\left(1+2 \eta^{2}\right) \operatorname{erfc} \eta-2 \pi^{-1 / 2} \eta e^{-\eta^{2}}\right]+2\left(\pi^{-1 / 2} e^{-\eta^{2}}-\eta \operatorname{erfc} \eta\right)^{2}$

The boundary condition that $f=0$ at $\eta=0$ and $\eta=\infty$ gives

$$
A=0, \quad B=2 / \pi
$$

The function $h$ is obtained by a quadrature of $f$, and the function $p$ by the double quadrature of $4 g^{2}$. The final analytic expressions for $f, h$ and $p$, are

$$
\begin{aligned}
& f=\frac{2}{\pi}\left[\left(1+2 \eta^{2}\right) \operatorname{erfc} \eta-2 \pi^{-1 / 2} \eta e^{-\eta^{2}}\right]-2\left(\pi^{-1 / 2} e^{-\eta^{2}}-\eta \operatorname{erfc} \eta\right)^{2} \\
& h=\frac{2}{3 \pi}\left[\left(3 \eta+2 \eta^{3}\right) \operatorname{erfc} \eta-2 \pi^{-1 / 2}\left(1+\eta^{2}\right) e^{-\eta^{2}}\right]-\frac{2 \eta}{3}\left(\pi^{-1 / 2} e^{-\eta^{2}}-\eta \operatorname{erfc} \eta\right)^{2} \\
& \quad-\frac{2}{3(\pi)^{1 / 2}} e^{-\eta^{2}} \operatorname{erfc} \eta+\frac{2(2)^{1 / 2}}{3(\pi)^{1 / 2}} \operatorname{erfc} 2^{1 / 2} \eta+\frac{2}{3(\pi)^{1 / 2}}\left(\frac{2}{\pi}-2^{1 / 2}+1\right) .
\end{aligned}
$$

${ }^{2}$ Goldstein, loc. cit. p. 183. A similar approximation has been made there.

${ }^{3}$ The author is indebted to the referee of this paper for the particular solution to Eq. (5). 


$$
\begin{aligned}
p=(1 & \left.+2 \eta^{2}\right)(\operatorname{erfc} \eta)^{2}-\frac{4 \eta}{\pi^{1 / 2}} e^{-\eta^{2}} \operatorname{erfc} \eta-\frac{2}{\pi} e^{-2 \eta^{2}}+\frac{4(2)^{1 / 2}}{\pi^{1 / 2}} \eta \operatorname{erfcc} 2^{1 / 2} \eta\left(-1+\frac{2}{\pi}\right) \\
& +\frac{4 \eta}{\pi^{1 / 2}}\left(\frac{2}{\pi}-2^{1 / 2}+1\right)+\text { const. }
\end{aligned}
$$

Note that there is an anomaly in the behaviour of the pressure which makes it approach infinity as $\eta \rightarrow \infty$ and precludes the specification of the constant in the last quadrature by a reasonable physical boundary condition. The anomaly is due to the acceleration of the infinite mass of fluid.

The functions $g, f$, and $h$ are given in Fig. 1 .

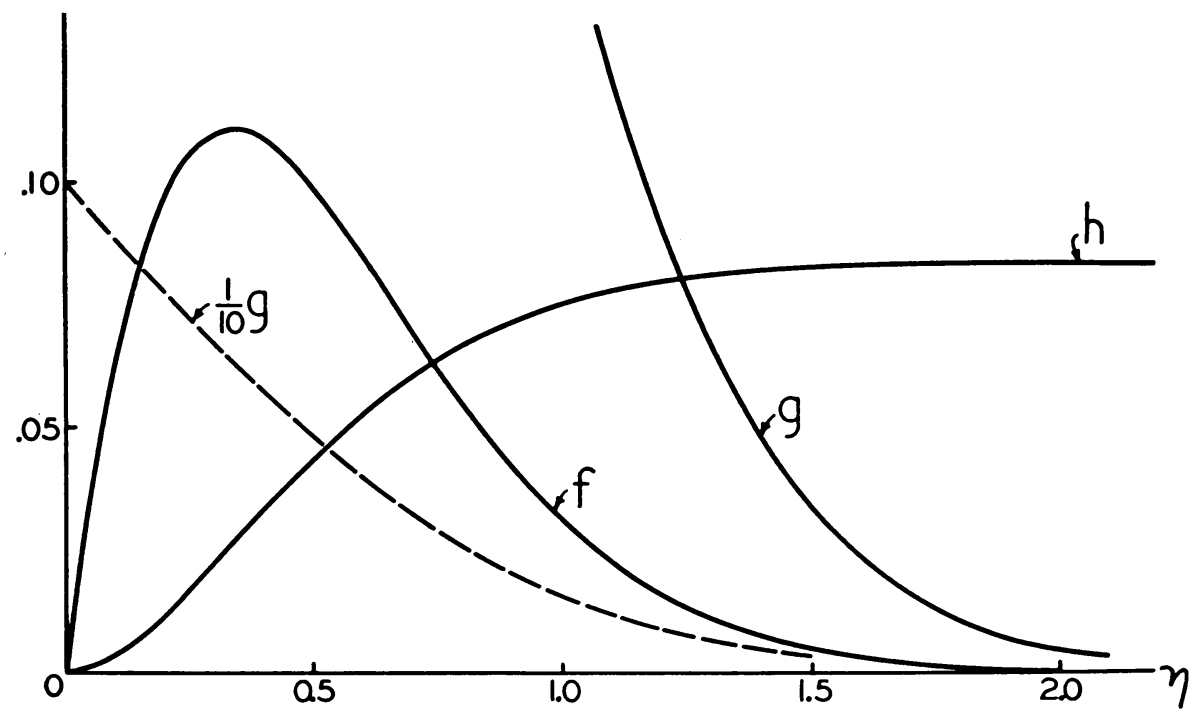

FIG. 1. The flow functions.

4. Stream function. A stream function may be defined by the equations

$$
\begin{aligned}
& u=-\frac{1}{r} \frac{\partial \psi}{\partial z}, \\
& w=\frac{1}{r} \frac{\partial \psi}{\partial r},
\end{aligned}
$$

whence the stream function may be expressed

$$
\psi=-2 \nu^{1 / 2} \Omega^{2} t^{3 / 2} r^{2} h(\eta) .
$$

This gives stream surfaces which are surfaces of revolution.

In conclusion I express a deep sense of gratitude to Prof. M. Ray, D.Sc. for his kind help in preparation of this note. 